\title{
Humanidades en interdisciplina
}

\author{
Daniela Soledad Gonzalez \\ gonzalezdanielasoledad@yahoo.com.ar \\ https://orcid.org/0000-0003-2437-531X \\ Facultad de Filosofía y Letras - Universidad Nacional de Cuyo (FFyL) \\ Mendoza, Argentina
}

Recibido: 25/05/2020 Aceptado: 22/10/2020

\begin{abstract}
Resumen
El presente artículo busca poner en valor los aportes de las Ciencias Humanas a la construcción interdisciplinar de conocimientos relacionados con problemáticas que exigen la aplicación urgente de nuevas estrategias de comprensión y acción como las siguientes: comportamientos sociales disfuncionales en las redes sociales, adicciones, trastornos de la alimentación, manipulación de la información en las redes y crisis de angustia existencial. Se argumenta que la inclusión o potenciación de la injerencia de las Humanidades en los estudios interdisciplinares orientados a prevenir la aparición o el agravamiento de dichas problemáticas y otras afines tiene como consecuencia mejores resultados. En primer lugar, se discute el significado de la interdisciplina y el papel de las Humanidades en ella. Luego, se presenta como ejemplo de inclusión de las Humanidades en los proyectos de intervención socio-cultural interdisciplinares un proyecto que se llevó a cabo en General Alvear (Mendoza, Argentina), cuya implementación se basó principalmente en una serie de talleres de reflexión en torno a diversos objetos culturales (publicidades, propagandas, humor gráfico, videos, etc.) dictados por investigadores de las diversas áreas del conocimiento. El trabajo se cierra con una conclusión.
\end{abstract}

Palabras clave: Interdisciplina. Humanidades. Salud. Ciudadanía.

\section{Humanidades na interdisciplina}

\section{Resumo}

Este artigo busca valorizar as contribuições das Ciências Humanas para a construção interdisciplinar de conhecimentos relacionados a problemas que requerem a aplicação urgente de novas estratégias de compreensão e ação, tais como: comportamentos sociais disfuncionais em redes sociais, dependências, distúrbios alimentares, manipulação de informação em redes e crise de angústia existencial. Argumenta-se que a inclusão ou potencialização da interferência das Ciências Humanas nos estudos interdisciplinares, visando prevenir o surgimento ou agravamento desses problemas e de outros problemas relacionados, tem como consequiência melhores resultados. Primeiro, são discutidos o significado da interdisciplina e o papel das Humanidades nela. Depois, um projeto que foi realizado em General Alvear (Mendoza, Argentina), cuja implementação se baseou principalmente em uma série de oficinas de reflexão em torno de vários objetos culturais (anúncios, propagandas, humor gráfico, vídeos, etc.) ditados por pesquisadores de diversas áreas do conhecimento, é apresentado como exemplo da inserção das Ciências Humanas em projetos de intervenção sociocultural interdisciplinar.O trabalho termina com uma conclusão.

Palavras chave: Interdisciplina. Humanidades. Saúde. Cidadania. 


\title{
Humanities in Interdiscipline
}

\begin{abstract}
This article aims to value the contributions of Human Sciences to the interdisciplinary construction of knowledge related to problems that require the urgent application of new strategies of understanding and action such as the following ones: dysfunctional social behaviors in social networks, addictions, disorders of food, information manipulation in networks and existential sadness crisis. It is argued that the inclusion or enhancement of the interference of the Humanities in interdisciplinary studies tending to prevent the occurrence or aggravation of such problems and other related ones produces better results. First, the meaning of interdiscipline and the role of Humanities in it are discussed. Then, a project that was carried out in General Alvear (Mendoza, Argentina) is presented as an example of the inclusion of Humanities in interdisciplinary social-cultural intervention projects. The implementation of this project was based, primarily, on a series of discussion workshops on various cultural objects (advertising, propaganda, humor, videos, etc.) dictated by researchers from various areas of knowledge. The work closes with a conclusion.
\end{abstract}

Keywords: Interdiscipline. Humanities. Health. Citizenship.

\section{Estado de la cuestión: interdisciplina y Humanidades}

En la actualidad, la sociedad demanda insistentemente la realización de proyectos de investigación interdisciplinarios y centrados en las habilidades sociales y cognitivas holísticas que permitan al individuo integrarse en un mundo globalizado, mediatizado y complejo, a la vez que le permitan desarrollarse como un ser único y diferente de los demás en diversos aspectos.

La creciente especialización de las diversas áreas del conocimiento ha llevado en muchos casos a la desconexión de dichas áreas e incluso al desmerecimiento por parte de ciertas comunidades científicas de los conocimientos que se manejan en otras disciplinas. Entre otras voces que se alzan en la actualidad para advertir sobre los peligros de este proceder, se encuentra el programa Intellectual Humility, que hace un llamamiento a cultivar la virtud intelectual de la humildad en orden a poder establecer un diálogo abierto con otros miembros de la comunidad científica que acepte el disenso y apunte a la construcción de conocimientos en conjunto. En efecto, como afirma Zygmunt Bauman, "El diálogo real no es hablar con gente que piensa lo mismo que tú" (Esparza, 2016). Es necesario superar "el egocentrismo intelectual y el hermetismo del pensamiento" (Carvajal Escobar, 2010: 162).

En esta línea, muchas universidades financian en la actualidad propuestas de diversa índole, que apuntan a la vinculación con el medio y a la construcción de conocimientos 
interdisciplinares. Un ejemplo de este tipo de iniciativas es el Programa de Nacionalización de la Formación Secundaria Argentina, destinado a incorporar en las instituciones secundarias argentinas saberes y valores sociales, ambientales y culturales. Otras propuestas que se dirigen al objetivo de orientar las ciencias humanas a la interdisciplina y aprovechar sus conocimientos para el tratamiento de problemáticas sociales en el ámbito de la salud y la convivencia ciudadana son las siguientes:

(a) La carrera de Especialización en Gestión de Proyectos Interdisciplinares en Contexto Social (GePICS), creada recientemente por la Universidad de Buenos Aires. Esta carrera ha sido diseñada para la formación de profesionales de las disciplinas proyectuales y sociales en la gestión de proyectos públicos y privados, incluyendo las variables socioculturales, políticas e históricas, económicas, tecnológicas y ambientales. En particular, la universidad es un ámbito propicio para el impulso de este tipo de iniciativas, que acerquen a todos los ámbitos de la sociedad la educación como bien público y gratuito y promuevan, de este modo, la inclusión y la igualdad de oportunidades para todos los ciudadanos.

(b) La Diplomatura en Suicidología de la Universidad de Flores, cuyo objetivo es aportar marcos teórico-metodológicos y éticos para el manejo y la intervención en procesos suicidas en diversos ámbitos y contextos.

(c) El proyecto I+D de la Universidad Complutense de Madrid denominado ALETHEIA, dedicado al Arteterapia como medio de elaboración de los traumas mediante el manejo de la memoria emocional. Está destinado a personas que han sufrido estrés postraumático y contempla una parte teórica junto con el desarrollo de herramientas metodológicas aplicables en situaciones de violencia con consecuencias traumáticas. Es interesante destacar el carácter estratégico de este plan para el desarrollo socio-comunitario. Sus autores lo explican de la siguiente manera: "La dimensión estética, artística, cultural de la vida social es una de las interfaces que pueden ser utilizadas para conectar a las instituciones, las políticas o los centros de decisión a nivel nacional/local europea con la vida social. En este sentido, nuestra propuesta se adapta tanto a la Estrategia Española "Estrategia Española de Ciencia, Tecnología e Innovación" como a la estrategia europea "Inclusive, Innovative and Reflective Societies", dentro de Horizon 2020" (Universidad Complutense de Madrid, 2016).

Este trabajo busca contribuir al ámbito científico de la interdisciplina revalorizando las Ciencias Humanas como elemento fundamental para la comprensión integral y para la acción 
preventiva y el tratamiento de problemáticas complejas que afectan a la salud y la convivencia de las personas. Como botón de muestra, se puede señalar la reciente firma de la Magna Charta Humanitatum en la Universidad de Salamanca (2018), un documento que defiende las Humanidades como disciplina transversal para todas las ciencias y para la sociedad en general e impulsa el conocimiento de las Ciencias Humanas y la realización de acciones para acercarla a la sociedad.

La construcción de saberes interdisciplinarios que alienten la adopción de conductas saludables y competencias lógico-discursivas críticas es hoy una urgencia, como observan numerosos autores en la actualidad (Carballeda, 2008; Carvajal Escobar, 2010; Kaufman, 2018; Manes, 2018; Gordillo, 2018; Barcia, citado por Solana, 2018). De hecho, una definición integral de la salud la concibe como el "estado de completo bienestar físico, mental y social" (OMS, 1948) y atender a la salud implica la consideración del estilo de vida de las personas y su situación sociocultural, entre otros aspectos (Junta de Andalucía, 2010).

Por otra parte, es sabido que diversos autores se han pronunciado acerca del estatuto epistemológico de la interdisciplina y han insistido en que no debe constituir una mera yuxtaposición de áreas del conocimiento. Por ello, en este plan se proyecta construir una interdisciplina real y funcional, i. e., un espacio de conceptualización y discusión conjunta entre las ramas del saber implicadas (Stolkiner, 1999, 2005; Follari, 2005; Carvajal Escobar, 2010; Pombo, 2013). "El paso de la multidisciplinariedad a la interdisciplinariedad y/o a la transdisciplinariedad (Posada, 2004; Max-Neef, 2005), requiere del desarrollo de metodologías de trabajo en equipo y de integración entre diferentes ciencias" (Carvajal Escobar, 2010: 158). Debido a que "una cooperación ocasional no es interdisciplina" (Elichiry, 1987: 337), es necesario dedicar tiempo a la constitución de equipos de trabajo, a la realización de reuniones entre los miembros del equipo e incluso a la redacción de material pedagógico y otros tipos de publicaciones en común, en orden a llegar al momento de la acción con conceptualizaciones comunes claras y enriquecidas.

\section{Análisis de caso: proyecto territorial UNCuyo 2018}

A continuación, se describe y analiza un proyecto de intervención sociocomunitaria llevado a cabo en General Alvear Mendoza, que fue financiado por la Universidad Nacional de Cuyo, cuyo título fue Talleres de reflexión y análisis de problemáticas socioculturales desde un 
enfoque interdisciplinar (Gonzalez y Benavides, 2018). La propuesta fue una de las que resultaron ganadoras en la I Convocatoria de Proyectos en el Territorio, organizada por la Coordinación de Desarrollo Territorial de la Universidad Nacional de Cuyo en el año 2018. Esta convocatoria tuvo como objetivo principal "fortalecer la política del desarrollo territorial de la Universidad considerando que la clave para lograr dicho fortalecimiento es la participación y representación de las Unidades Académicas de la UNCuyo" (Universidad Nacional de Cuyo, 2018b).

Para comenzar, conviene explicitar algunos aspectos metodológicos. Debido a que este plan tuvo una orientación hacia la aplicación de saberes y un enfoque cualitativo, resulta difícil hablar de hipótesis como una aseveración que deba ser demostrada concluyentemente (Hernández Sampieri, Fernández Collado y Baptista Lucio, 2006: 533). Se prefiere hablar de "planteamiento inicial" o "idea motor" del trabajo. La idea motor del proyecto fue la siguiente: las Ciencias Humanas puestas en interdisciplina con otras áreas del conocimiento, incluso las más exactas, pueden aportar herramientas para interpretar problemáticas individuales y sociales del ser humano en la actualidad y abordarlas con mayores probabilidades de éxito.

Los objetivos generales del plan fueron: (a) promover la construcción de conocimientos interdisciplinarios y significativos para la comunidad de General Alvear (Mendoza), (b) formar recursos humanos que aporten beneficios a la vida ciudadana y a la salud pública, (c) facilitar la articulación de emprendimientos de profesionales de la universidad con los de otras universidades e instituciones y (d) fomentar la participación de los distintos claustros en la formulación y ejecución de un proyecto de intervención sociocomunitaria.

Los objetivos específicos fueron (a) analizar en qué consisten los objetos culturales y de qué modo operan constantemente en la sociedad, (b) obtener herramientas para responder a las demandas individuales, sociales, políticas y culturales que estos objetos vehiculizan y (c) prevenir conductas alienantes y actitudes que dejan indefensos o sin capacidad de resolución de conflictos a los afectados por problemáticas como comportamientos sociales disfuncionales relacionados con el uso de las redes sociales, discriminación, micromachismos y machismos explícitos, malas prácticas de alimentación y de manipulación de alimentos, adicciones, transtornos de la alimentación (bulimia y anorexia), angustia existencial, entre otras.

El primer aspecto destacable del proyecto es su carácter interdisciplinario. En orden a conseguir una visión holística de los complejos problemas que enfrenta la sociedad actual y 
proponer acciones que tiendan a disminuirlos, es necesario un enfoque interdisciplinario de abordaje. Como señala Carvajal Escobar, "Los problemas fundamentales que enfrenta la humanidad, obligan a estudiarlos como un todo, demandando el concurso de todas las potencialidades del conocimiento humano, y exigiendo enfocarlos como complejos, inseparables y retroalimentados; de tal forma que surge la necesidad de abordar una visión integral e interdisciplinaria para resolverlos" (Carvajal Escobar, 2010: 165). Esta particularidad del plan de trabajo que se presenta aquí le otorgó originalidad a la propuesta.

El plan de trabajo tendió tanto al avance en el conocimiento como a una aplicación práctica. Pretendió intervenir en una comunidad en orden a propiciar cambios en ella; al mismo tiempo, buscó indagar conocimientos disciplinares y generar teoría sobre una metodología interdisciplinar que favorezca el empoderamiento de los ciudadanos. Por ello, la investigación que se planteó puede etiquetarse como “investigación-acción” (Blández Ángel, 2000; Hernández Sampieri, Fernández Collado y Baptista Lucio, 2006; Anderson y Herr, 2007; Sirvent, 2010). Como señala Stolkiner (2005: 5-6), la interdisciplina es, a la vez, ciencia y práctica. Por otra parte, Stolkiner (2005: 5) afirma que "hay puntos de confluencia entre el enfoque interdisciplinario y las estrategias cualitativas de investigación". Por ende, es posible caracterizar la investigación realizada como cualitativa.

En cuanto al trabajo de campo que se llevó a cabo, se trató del dictado de talleres por parte de un grupo de investigadores en diversas disciplinas, como la Filosofía, las Letras, la Psicología, las Ciencias de la Educación, la Danza, la Educación Física, la Gestión Ambiental y la Industria de la Alimentación. El modo de trabajo en cada taller consistió en el uso de objetos culturales como disparadores de lluvias de ideas y el diálogo con los participantes para conocer sus conocimientos previos, en orden a establecer un aprendizaje significativo. Al final de cada taller, se hicieron preguntas destinadas a la metacognición y se incentivó la puesta en común de ideas afines. A través de la reflexión orientada, se tendió a construir colectivamente un saber teórico-práctico que aportara herramientas para una vida ciudadana enriquecedora en un mundo cambiante y complejo como el de hoy. Desde la interdisciplinariedad se esperó alcanzar una visión enriquecedora y abarcadora de los fenómenos en cuestión.

Como el proyecto se orientó a promover el abordaje integral del ser humano, los ejes temáticos sobre los que se trabajó, en diferentes niveles, fueron los siguientes: la promoción de la salud, la participación ciudadana, la mejora de la calidad de vida, la gestión cultural, la 
divulgación científica, la responsabilidad social, el arte como herramienta de inclusión social y las nuevas pedagogías y herramientas actuales en la educación.

En cuanto a los resultados del proyecto, uno de ellos fue la publicación de un libro que contiene los contenidos interdisciplinares básicos elaborados por el equipo de investigación (Gonzalez, 2018) y la elaboración de diversos materiales audiovisuales, los cuales se utilizaron en cada uno de los talleres. Otro resultado fue, por supuesto, la realización del ciclo de talleres. A continuación, se detallan los talleres dictados, sus fechas y quiénes los dictaron:

Tabla 1 - Detalle de los talleres dictados en el ciclo

\begin{tabular}{|c|c|}
\hline Fecha & Talleres y encargados \\
\hline $21 / 07 / 2018$ & $\begin{array}{c}\text { Primer encuentro de talleres de capacitación } \\
\text { - Taller "Análisis de los discursos actuales que circulan en las redes sociales y de su impacto" - } \\
\text { Daniela Soledad Gonzalez } \\
\text { - Taller "Desigualdad, machismo y micromachismos en las redes sociales" - Victoria Lucero } \\
\text { - Taller "Estrategias lingüísticas para leer en internet en la era de la posverdad" - Irene Herrera } \\
\text { Volpe } \\
\text { - Taller "Asertividad y cortesía. Estrategias para un debate constructivo en las redes sociales" - } \\
\text { Ana Ruth Enriques } \\
\text { - Taller "El sentido y el uso de la historia" - Victoria Sabrina Piseghelli }\end{array}$ \\
\hline $04 / 08 / 2018$ & $\begin{array}{c}\text { Segundo encuentro de talleres de capacitación } \\
\text { - Taller "Conócete a ti mismo: la importancia del autoconocimiento para la consecución de una } \\
\text { vida plena" - Cristian Eduardo Benavides } \\
\text { - Taller "El arte como medio de conexión vital con lo sublime" - Juan Francisco López } \\
\text { - Taller "Desafíos actuales de la educación" - María Elisa Di Marco }\end{array}$ \\
\hline $18 / 08 / 2018$ & $\begin{array}{c}\text { Tercer encuentro de talleres de capacitación } \\
\text { - Taller "Mejora de las habilidades sociales para la prevención de adicciones y trastornos de la } \\
\text { alimentación" - María Lourdes Barrenechea } \\
\text { - Taller "Calidad de vida, alimentación y manipulación de alimentos" - Mariana Eugenia } \\
\text { Noguerol } \\
\text { - Taller "Los beneficios de mantener el cuerpo en movimiento" - Fernando Gabriel Gonzalez } \\
\text { - Taller "La danza como medio para llevar una vida saludable" - Juan Pablo Guerrera }\end{array}$ \\
\hline $01 / 09 / 2018$ & $\begin{array}{c}\text { Cuarto encuentro de talleres de capacitación } \\
\text { - Taller "La importancia de descubrir } \\
\text { las metáforas que operan en los discursos cotidianos" - Daniela Soledad Gonzalez } \\
\text { - Taller "El desarrollo sustentable de las } \\
\text { actividades" - Gabriela Susana Donaire y Silvina López }\end{array}$ \\
\hline $15 / 09 / 2018$ & Actividad evaluativa final (muestra) \\
\hline
\end{tabular}

Se llevó a cabo, además, una muestra final, en la que cada participante presentó el producto de sus reflexiones y análisis, que incluyó videos, afiches, folletos, presentaciones en 
Power Point o Prezi y producciones escritas acerca de las temáticas de su interés, elegidas de entre las expuestas a lo largo del ciclo o relacionadas con ellas. Por último, el presente artículo es también un resultado del proyecto.

La actividad fue sumamente enriquecedora tanto para los asistentes como para los capacitadores. Se formó un grupo de profesionales y alumnos estrechamente unidos y entusiasmados. Los asistentes al curso participaron activamente, compartieron experiencias personales y agradecieron el haber recibido los talleres. Algunas de sus valoraciones se copiaron de WhatsApp y se adjuntan en el anexo con el registro audiovisual de las actividades. Se nos solicitó hacer una nueva propuesta para continuar con el diálogo profundo entablado.

En cuanto a los resultados a largo plazo del plan, se apuntó a vincular el trabajo de la universidad con el medio inaugurando un espacio de trabajo con la Dirección de Cultura de General Alvear (Mendoza), que se puede continuar enriqueciendo a futuro. La vinculación con el medio y el trabajo interdisciplinar para la promoción de la salud tienden a generar como resultado remoto un empoderamiento de los ciudadanos que les permita desarrollarse como personas plenas e integrarse mejor a la sociedad.

En adición, con el presente proyecto se beneficiaron los alumnos que participan de él como miembros. Además, obtendrán provecho de las actividades los alumnos que cursen en las cátedras en las que dictan clases los docentes que conforman el equipo, puesto que se mejorará los contenidos que se impartan gracias a la experiencia tanto de investigación como de acción planificadas.

\section{Consideraciones finales}

Cada vez se hace más patente la necesidad de la construcción interdisciplinar de conocimientos en los ámbitos de la promoción de la salud y la ciudadanía responsable. En este trabajo, se ha intentado valorizar las contribuciones de las Humanidades para buscar estrategias de intervención sobre algunas problemáticas ubicadas dentro de estos ámbitos, como la falta de asertividad y cortesía en las redes sociales, la incentivación de la desigualdad y el machismo, la estimulación de cánones de belleza que conduce a muchas personas a trastornos de la alimentación, la falsificación o manipulación de la información en las redes sociales y la gran cantidad de crisis emocionales y afectivas. Se sostiene que es necesario incluir o aumentar la 
injerencia de las humanidades en los estudios interdisciplinares orientados a prevenir la aparición o el agravamiento de dichas problemáticas y otras afines.

En primer lugar, se precisó que la interdisciplina no es una mera yuxtaposición de disciplinas diversas, sino que debe constituir un espacio de construcción de conocimientos en conjunto y debe plantearse como una discusión continua constante entre distintas ramas del saber implicadas. Por ello, es necesario constituir equipos de trabajo, realizar reuniones entre los miembros del equipo y preparar material pedagógico en conjunto para llegar al momento de la intervención sociocultural con conceptualizaciones comunes claras y enriquecidas. Sobre el papel de las Humanidades en los estudios y proyectos interdisciplinares, se insistió en que debe dárseles un papel más activo en ámbitos como el de la salud y la ciudadanía responsable, en los que suelen cumplir un papel secundario.

Para ejemplificar cómo puede llevarse a cabo la inclusión de las humanidades en los estudios y proyectos de intervención socio-comunitaria interdisciplinares, se describió y analizó un proyecto que se llevó a cabo en General Alvear (Mendoza, Argentina), cuya implementación se basó principalmente en una serie de talleres de reflexión en torno a diversos objetos culturales dictados por investigadores de las siguientes áreas: Filosofía, Letras, Psicología, Ciencias de la Educación, Danza, Educación Física, Gestión Ambiental e Industria de la Alimentación. En este proyecto se apuntó hacia una toma de conciencia sobre el poder del lenguaje performativo, la acción sutil de ciertos discursos recibidos acríticamente, el estrés como síntoma del estilo de vida actual y los mandatos sociales de las modas. Las reflexiones se orientaron, sobre todo, al desarrollo de habilidades sociales (autoconocimiento, autoestima, empatía, autocontrol, asertividad, habilidad para tomar decisiones, resiliencia, etc.).

Como resultado próximo del proyecto, se realizó una muestra final en la que los asistentes expusieron una temática de su interés. Otro resultado destacable es la formación de recursos humanos, el enriquecimiento que los mismos miembros del proyecto obtuvieron de la experiencia. Como resultado remoto, se espera haber realizado un aporte al empoderamiento de los ciudadanos que les permita desarrollarse como personas plenas e integrarse mejor a la sociedad. En un futuro, es posible redirigir las actividades hacia ámbitos diferentes del lugar de origen del proyecto. También es posible que se realice una adaptación de estos conocimientos a algún formato audiovisual de circulación virtual (posiblemente, uno o varios videos de YouTube). 


\section{Referencias}

ANDERSON, G.; HERR, K. Teacher Research: Action Research as a Valid Form of Knowledge Generation.). En: I. Sverdlick (Ed.), La investigación educativa: Una herramienta de conocimiento y de acción. Buenos Aires: Noveduc, 2007.

BLÁNDEZ, Ángel, J. La investigación-acción: un reto para el profesorado: Guía práctica para grupos de trabajo, seminarios y grupos de investigación, 2. ${ }^{a}$ ed., Barcelona: INDE, 2000.

CARBALLEDA, J. La interdisciplina como diálogo. Una visión desde el campo de la salud. Trabajo social y salud, n. 61, 121-126, 2008.

CARVAJAL ESCOBAR, Y. Interdisciplinariedad: Desafío para la educación superior y la investigación. Revista Luna Azul, n. 31, 156-169, 2010.

ELICHIRY, N. (Comp.). El niño y la escuela. Reflexiones sobre lo obvio. Bs As: Ed. Nueva Visión, 1987.

ESPARZA, D. Zygmunt Bauman: "El diálogo real no es hablar con gente que piensa lo mismo que tú". Aleteia, 7 de septiembre de 2016. Disponible en: https://es.aleteia.org/2016/09/07/zygmunt-bauman-el-dialogo-real-no-es-hablar-congente-que-piensa-lo-mismo-que-tu/ (Acceso en: 22 nov. 2018).

FOLLARI, R. La interdisciplina revisitada. Andamios, v. 1, n. 2, 7-17, 2005.

GONZALEZ, D. (ed.). Análisis interdisciplinar de objetos culturales sobre problemáticas sociales, GRIN, München, 2018, pp. 36-48, 2018.

GONZALEZ, D.; BENAVIDES, C. Talleres de reflexión y análisis de problemáticas socioculturales desde un enfoque interdisciplinar. Proyecto territorial UNCuyo 2018. Mendoza: Universidad Nacional de Cuyo, 2018.

GORDILLO, V. Uno de cada cinco jóvenes no tiene quien lo aconseje. Unidiversidad, 23 de noviembre de 2018. Disponible en: http://www.unidiversidad.com.ar/el-225-de-losjovenes-no-tiene-quien-loaconseje?fbclid=IwAR1ZrLecqtBwNd2QQfINczPUjvle5eskfFui_x84bd4Ggn5Mz9Ov22mDbc (Acceso en: 28 nov. 2018).

HERNÁNDEZ SAMPIERI, R.; FERNÁNDEZ COLLADO, C.; BAPTISTA LUCIO, P. Metodología de la investigación, 4. ${ }^{\mathrm{a}}$ ed., México: McGrawHill, 2006.

JUNTA DE ANDALUCÍA. La promoción de la salud como derecho, 2010. Disponible en: https://anteproyectoleysaludpublicadeandalucia.wordpress.com/2010/12/09/lapromocion-de-la-salud-como-derecho/ (Acceso en: 23 nov. 2018).

KAUFMAN, S. Do You Have a Healthy Personality? Scientific American, 15 de noviembre de 2018. Disponible en: https://blogs.scientificamerican.com/beautiful-minds/do-youhave-a-healthy-personality/ (Acceso en: 15 nov. 2018).

MANES, F. La soledad como cuestión de Estado. Perfil, 18 de marzo de 2018. Disponible en: https://www.perfil.com/noticias/columnistas/la-soledad-como-cuestion-deestado.phtml (Acceso en: 18 mar. 2018).

ORGANIZACIÓN MUNDIAL DE LA SALUD. Constitución de la OMS de la salud, 1948. Disponible en: https://www.who.int/about/mission/es/ (Acceso en: 23 nov. 2018).

POMBO, O. Epistemología de la interdisciplinariedad. La construcción de un nuevo modelo de comprensión. Interdisciplina, v. 1, n. 1, 21-49, 2013.

SIRVENT, M. La investigación acción participativa y la animación socio-cultural. Su papel en la participación ciudadana. TPS, n. 1, 61-74, 2010. 
SOLANA, I. Educación del carácter para prevenir conductas nocivas entre los jóvenes como el consumismo y las adicciones digitales. Vida Universitaria, 20 de junio de 2018. Disponible en: https://www.unav.edu/web/vidauniversitaria/detallenoticiapestania/2018/06/20/educacion-del-caracter-para-prevenirconductas-nocivas-entre-los-jovenes-como-el-consumismo-y-las-adiccionesdigitales?articleId=18418612 (Acceso en: 20 jun. 2018).

STOLKINER, A. La interdisciplina: entre la epistemología y las prácticas. Revista Campo PsiRevista de Información especializada, v. 3, n. 10, 1999.

STOLKINER, A. Interdisciplina y salud mental. En: IX Jornadas Nacionales de Salud Mental, I Jornadas Provinciales de Psicología, Salud Mental y Mundialización: Estrategias posibles en la Argentina de hoy, 7 y 8 de octubre 2005, Posadas (Misiones, Argentina).

Disponible

en:

http://www.psi.uba.ar/academica/carrerasdegrado/psicologia/sitios_catedras/obligatoria s/066_salud2/material/unidad1/subunidad_1_3/stolkiner_interdisciplina_salud_mental. pdf (Acceso en: 23 nov. 2018).

UNIVERSIDAD COMPLUTENSE DE MADRID. ALETHEIA: Arte, Arteterapia, Trauma y memoria emocional. I+D, 2016. Disponible en: https://www.ucm.es/aletheia/noticias/surge-el-proyectoaletheia?fbclid=IwAR3jrATVQmJnh9UbHilQ7ukWLcHLW41dwXtAWicPQbxob14i nYNbAmyIDP0 (Acceso en : 27 nov. 2018).

UNIVERSIDAD DE BUENOS AIRES. Carrera de Especialización en Gestión de Proyectos Interdisciplinares en Contexto Social, 2018. Disponible en: http://www.uba.ar/posgrados/archivos/CE_Gestion\%20ProyecInterdi\%20Contexto\%20 Social.pdf (Acceso en: 22 nov. 2018).

UNIVERSIDAD DE FLORES. Diplomatura en suicidología, 2018. Disponible en http://www.uflo.edu.ar/diplomaturas/4-diplomatura-en-suicidologaa (Acceso en: 22 nov. 2018).

UNIVERSIDAD DE SALAMANCA. Salamanca defiende a las Humanidades con la firma de la Magna Charta Humanitatum, 2018. Disponible en: https://www.tribunasalamanca.com/noticias/salamanca-defiende-a-las-humanidadescon-la-firma-de-la-magna-charta-humanitatum (Acceso en: 28 nov. 2018).

UNIVERSIDAD NACIONAL DE CUYO. La UNCuyo busca fortalecer la educación secundaria, 2018a Disponible en: http://www.uncuyo.edu.ar/academica/la-uncuyobusca-fortalecer-la-educacionsecundaria?fbclid=IwAR1ok_xBCoR4d17aHFsVfmt4DfqVT7ESrjF1T7mB7ejDgqibp9dU3T4jW0 (Acceso en: 23 nov. 2018).

UNIVERSIDAD NACIONAL DE CUYO. Acta de selección de proyectos I Convocatoria de proyectos en el territorio, 2018b. Disponible en: http://www.uncuyo.edu.ar/desarrollo/upload/acta-de-seleccion-de-proyectos.pdf (Acceso en: 05 oct. 2018).

Autora

Daniela Soledad Gonzalez (e-mail: gonzalezdanielasoledad@yahoo.com.ar, ORCID id: https://orcid.org/0000-0003-2437-531X) es Doctora en Letras, Profesora de grado universitario en Lengua y Literatura y Licenciada en Letras por la Facultad de Filosofía y Letras de la Universidad Nacional de Cuyo (FFyL-UNCuyo). Es becaria de investigación posdoctoral del 
CONICET. Se desempeña como Profesora Adjunta en la FFyL-UNCuyo. Ha realizado diversas publicaciones de nivel internacional sobre variados temas lingüísticos, entre los que se encuentran los adverbios periféricos, la metáfora y la metonimia conceptual, el embodiment, el lunfardo, los eufemismos y disfemismos, las funciones discursivas de la nominalización y la escritura científica. Ha participado en numerosos proyectos de investigación. Es miembro del Instituto de Lingüística Joan Corominas (UNCuyo) y de otras asociaciones de ciencia y técnica como el Centro de Investigaciones Cuyo, el Centro de Estudios de Filosofía Clásica (CEFIC, UNCuyo) y el Instituto de Filosofía (UNCuyo). Forma parte del Comité Editorial de la revista Anales de Lingüística (ISSN: 0325-3597) y del Comité Científico de la revista PIRQAS (ISSN: 2684-0332). Es evaluadora para diversas revistas científicas.

\section{Como citar el artículo:}

Gonzalez, Daniela Soledad. Humanidades en interdisciplina. Revista Paradigma Vol. XLII, Nro. 2, Diciembre de 2021 / 375 - 386.

DOI: https://doi.org/10.37618/PARADIGMA.1011-2251.2021.p375-386.id897 